\title{
Resource Utilization Among Glaucoma Patients in the UK Treated with Beta-Blocker and Non-Beta- Blocker Adjunctive Therapy: A Retrospective Cohort Analysis
}

\author{
Ananth Viswanathan · Claudio Spera · Anmol Mullins • \\ David Covert · Judit Banhazi · Paul McDwyer · Eibhlin Hudson • \\ Alberto Ferreira
}

Received: March 15, 2017 / Published online: May 30, 2017

(c) The Author(s) 2017. This article is an open access publication

\section{ABSTRACT}

Introduction: Few studies have examined outcomes and potential complications among glaucoma patients who are prescribed topical beta-blockers. This study examined resource usage (number of GP visits and hospitalizations) and diagnoses of respiratory or cardiovascular conditions among glaucoma patients prescribed beta-blockers compared to patients not prescribed beta-blockers.

Methods: A retrospective cohort analysis was conducted using data from the UK Clinical

Enhanced content To view enhanced content for this article go to http://www.medengine.com/Redeem/ 3A18F06041CF66EC.

Electronic supplementary material The online version of this article (doi:10.1007/s12325-017-0541-8) contains supplementary material, which is available to authorized users.

A. Viswanathan

NIHR Biomedical Research Centre, UCL Institute of Ophthalmology, Moorfields Eye Hospital NHSFT, London, UK

C. Spera · A. Ferreira

Novartis Pharma AG, Basel, Switzerland

A. Mullins - D. Covert

Novartis Pharmaceuticals Corporation, East

Hanover, NJ, USA

J. Banhazi $(\varangle) \cdot$ P. McDwyer · E. Hudson Novartis Ireland Limited, Dublin, Ireland e-mail: judit.banhazi@novartis.com
Practice Research Datalink (CPRD) database over the period January 1, 2006 to March 31, 2014. Adult patients with at least one diagnosis of glaucoma were categorized into beta-blocker users and non-beta-blocker users. Beta-blocker users were further separated into patients that maintained beta-blocker therapy and patients that discontinued beta-blocker treatment in year 2 of the post-index period. The CPRD data was queried directly to obtain the number of GP visits, and hospitalizations were extracted by linking the CPRD and Hospital Episode Statistics (HES) patient-level data.

Results: In the 12 months after being prescribed beta-blockers, patients that later discontinued beta-blocker treatment had a significantly higher average number of hospitalizations than patients that maintained beta-blocker therapy and the non-beta-blocker users $(p<0.05)$. In the year after beta-blocker initiation, there was a statistically significant within-group difference pre- and post-betablocker initiation for all groups, but the greatest number of GP visits occurred in the patients that subsequently discontinued beta-blocker treatment (mean 19.27). Patients that discontinued beta-blocker treatment were significantly more likely to have cardiovascular events than non-beta-blocker users in the post-index period $(p<0.05)$.

Conclusion: This study suggests that the introduction of beta-blockers in a certain group of patients who later discontinue their use is 
associated with increased use of medical resources (higher number of GP visits and hospitalizations) in glaucoma patients in the UK, which may be indicative of a potential relationship between use of topical beta-blockers in glaucoma therapy and adverse outcomes.

Keywords: Beta-blocker; CPRD; Glaucoma; Ophthalmology; Resource utilization

\section{INTRODUCTION}

Glaucoma is among the leading causes of irreversible blindness and represents a significant public health burden in countries with an aging population [1]. Worldwide, the total number of suspected cases of glaucoma is estimated to be over 60 million [2]. In the UK, glaucoma is estimated to affect $0.9 \%$ of the population and remains the second leading cause of blind registrations [3].

The most common type of glaucoma is primary open angle glaucoma (POAG), representing $90 \%$ of all glaucoma cases [4]. POAG is characterized by a progressive, chronic optic neuropathy. Elevated intraocular pressure (IOP) associated with the majority of POAG patients is related to a chronic progressive resistance of aqueous humor drainage through the trabecular meshwork in the anterior chamber of the eye [5]. Elevated IOP is a key risk factor for glaucoma onset and progression and is currently the only known modifiable risk factor.

A range of topical IOP-lowering agents, including prostaglandin analogues, selective or non-selective beta-blockers, selective adrenergic agonists, carbonic anhydrase inhibitors, and parasympathomimetics are currently licensed for use in glaucoma [6]. Guidelines published by the National Institute for Health and Clinical Excellence (NICE) recommend topical ocular hypotensive agents as the first-line treatment for glaucoma in patients who are at risk of vision loss within their lifetime; however, no agent is specifically prioritized [7]. Prescribing patterns in the UK do not indicate a clear preference for any single therapy though trends in treatment denote a notable shift from beta-blockers (BBs) to prostaglandin analogues for first-line use $[8,9]$. The use of adjunctive therapy, either in a fixed-dose combination or as separate medications, is common with almost $40 \%$ of PA patients requiring two or more medications within 5 years of diagnosis [9].

Despite contraindications, BBs are used in a significant number of patients with diagnoses of respiratory or cardiovascular conditions $[10,11]$. Few studies have examined the outcomes of glaucoma patients with contraindications for topical beta-blockers but who still receive them. Houde et al. [10] found that more than half of glaucoma patients treated with topical beta-blockers had a contraindication for their use, while Vinker et al. [11] found that more than $60 \%$ of patients with glaucoma and obstructive lung disease were using topical beta-blockers. Another study reported that up to $25 \%$ of glaucoma patients were receiving prescriptions for treatment of respiratory disease or congestive heart failure. Schmier et al. [12] found that the introduction of timolol, a topical beta-blocker, in glaucoma patients who later discontinue this treatment was associated with increased use of medical resources and higher payments; however, these results have not been confirmed in other clinical settings.

The primary objective of this study was to examine resource utilization (number of GP visits and hospitalizations) among patients prescribed BBs compared to patients not prescribed BBs in the UK clinical setting. For this purpose, data from the Clinical Practice Research Datalink (CPRD) and Hospital Episode Statistics (HES) databases were used. The CPRD in the UK includes anonymized longitudinal medical records for patients followed in primary care, and includes about $8 \%$ of the UK population with approximately 14 million patients, with around 5.4 million of these being currently alive and registered from 680 primary care practices spread throughout the UK [13]. Records are derived directly from GP software system and contain complete prescribing and diagnostic and clinical information with standard coding as well as information on tests requested, laboratory results, and referrals made at or following on from each consultation. Read codes are used to record symptoms, diagnoses, 
and procedures. The HES data contains records of all patients admitted to NHS hospitals in England. It contains details of inpatient care, outpatient appointments, and accident and emergency attendance records. Each HES record contains a wide range of information about an individual patient admitted to an NHS hospital, including clinical information about diagnoses and operations, patient demographics, and administrative information.

\section{METHODS}

The CPRD database was used to identify adult ( $\geq 18$ years of age) patients with at least one glaucoma diagnosis during the study identification period (January 01, 2006-March 31, 2011) and aligned with standard inclusion/exclusion criteria for retrospective research (see supplementary material for a complete list of criteria).

All patients were followed for 3 years. The year of 2011 was used as the limit for the identification period in order to allow 3 years follow-up for all patients.

Patients were categorized into two mutually exclusive cohorts (Fig. 1):

BB users

Based on a prescription of a free (or fixed) combination of a $\mathrm{BB}$ and a non-BB glaucoma treatment, at least once, in year 2 of the study but with no $\mathrm{BB}$ prescription in year 1 .

Non-BB users Patients with no prescription of BBs during the 3 years of follow-up, but treated with other glaucoma treatments (as per inclusion criteria).

There was a group of 'Other users': patients who were not eligible for the two groups. For example, patients who were prescribed a BB but not concomitantly with a non-BB glaucoma treatment or in a fixed combination. These patients were not analyzed.

The BB user cohort was further separated into maintainers (patients with prescriptions for BB treatment in year 2 and year 3) and discontinuers (patients with prescriptions for BB treatment in year 2 but not in year 3).

The start of the follow-up was the first date when a glaucoma diagnosis was recorded in the identification period (glaucoma diagnosis date). The index date depended on cohort assignment, being the date of first BB prescription in the follow-up period for the BB group and day 1 of year 2 for the non-BB cohort. All patients in the cohorts had 1 year of pre-index data (year 1 ) and 2 years of post-index data (year 2 and 3). The pre-index period was the time period from the glaucoma diagnosis date until (but not including) the index date, and the post-index period was the time period from the index date until the end of follow-up (end of year 3) (Fig. 2).

The event rates and resource use were analyzed for the first year of the post-index period, i.e., after initiating BBs in the BB user group. Discontinuation or maintenance on therapy was assessed in the second year of the post-index period.

The CPRD database was queried to obtain the number of GP visits, while hospitalizations were extracted by linking the CPRD and HES patient-level data. CPRD has full access to HES data, although not all patients in CPRD have HES linkage. The amount of overlap identified for this study was $66.1 \%$.

Respiratory and cardiovascular conditions were examined using all relevant respiratory and cardiovascular Read codes (patients having one or more respiratory or cardiovascular condition in the relevant periods). Codes sets were defined by an expert on Read coding and data extraction who defined the most appropriate lists of codes used at CPRD that would best describe cardiovascular and respiratory conditions comparing beta-blocker usage and nonuse in glaucoma patients in the UK. A total of 451 Read codes were selected for respiratory and 1369 codes were selected for cardiovascular disease.

Costing data from HES hospitalization codes were grouped using the Healthcare Resource Groups (HRG) grouper tool and appropriate costs were assigned using standard CPRD procedures. 


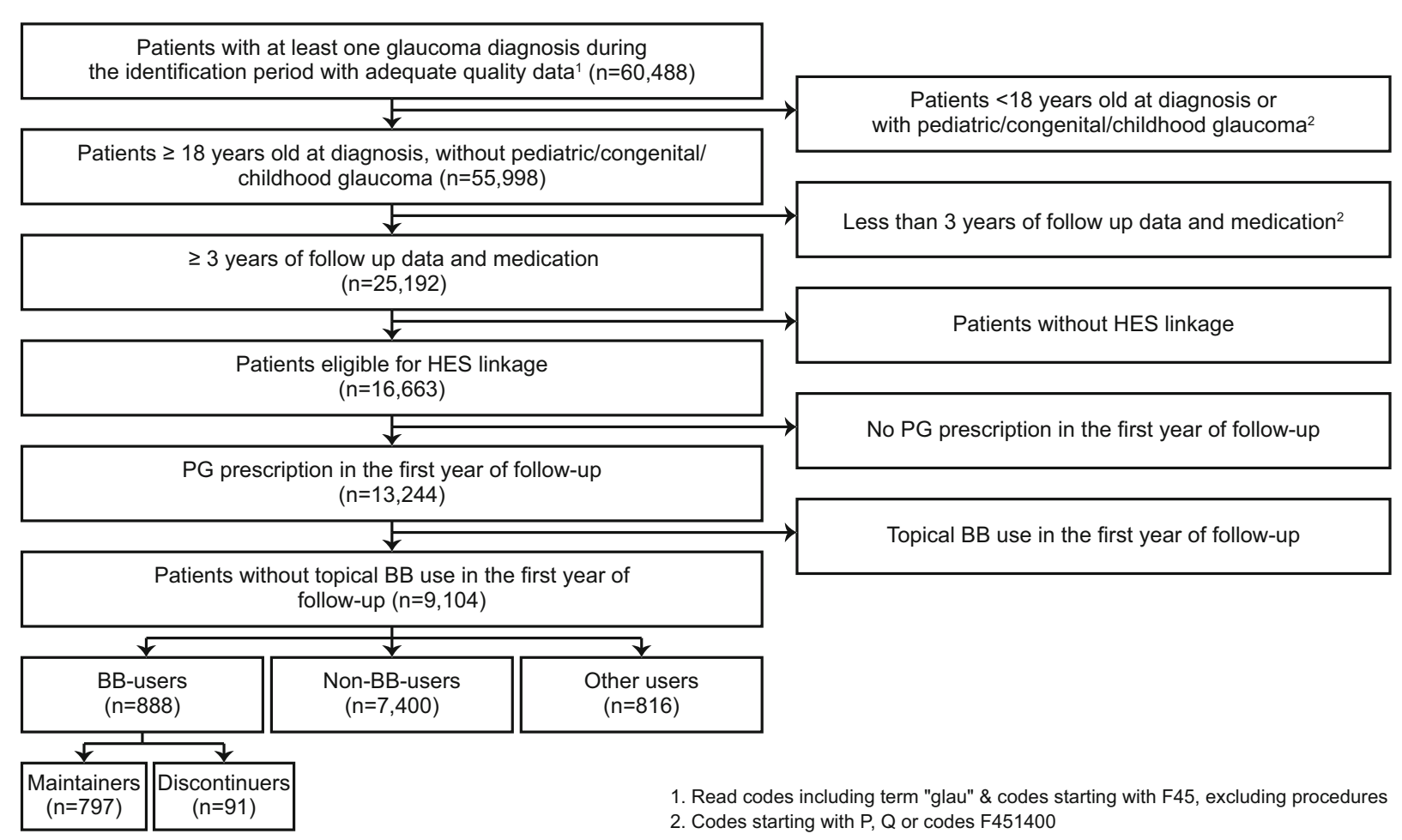

Fig. 1 Patient selection criteria

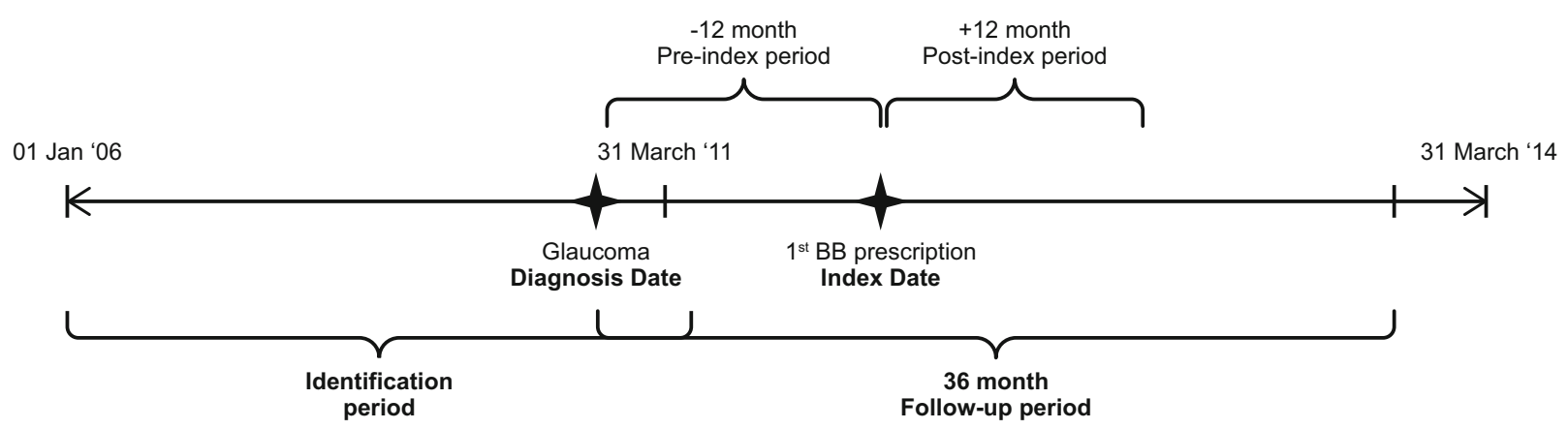

Fig. 2 Study time frames

\section{Statistical Analyses}

In addition to summary statistics, the Wilcoxon signed rank sum test was used for the pre- versus post-index comparisons of resource use within the individual treatment groups. Chi square tests were used for between-group comparison of event rates in the pre-index and the post-index period. McNemar's test (comparing correlated/dependent proportions) was used for the pre- versus post-index comparisons of event rates within the individual treatment groups.

A logistic regression model was developed to examine the presence of a cardiovascular condition in the 1-year post-index period and a separate logistic regression model was developed to examine the presence of a respiratory condition in the 1-year post-index period. The model was adjusted for BB use as well as age (at index), male [gender with reference female], 
region (London as reference category), any Charlson comorbidity (pre-index), and post-index prostaglandin use.

A negative binomial regression model was used to examine the association between BB use and resource use (i.e., number of hospitalizations and GP visits) controlling for confounding variables as described above.

This was a retrospective, non-interventional study: it is an analysis of existing data and does not contain any new studies with human or animal subjects performed by any of the authors.

\section{RESULTS}

\section{Patient Disposition}

A total of 9104 patients met the inclusion and exclusion criteria for the study (Fig. 1). Of these, 888 were BB users and 7400 were non-BB users. There were 816 'Other users' as defined in the "Methods". Of the BB users, patients that maintained $\mathrm{BB}$ use represented approximately $90 \%(n=797)$ and patients that discontinued BB use represented approximately 10\% $(n=91)$ of the cohort.

BB users had a mean age of 69.4 years compared to 70.7 years in the non-BB cohort. The cohorts had similar gender distributions, with male patients making up $49.5 \%$ and $47.3 \%$ of the $\mathrm{BB}$ cohort and non-BB cohort respectively (Table 1).

The Charlson comorbidity index (CCI) for each patient was calculated using the Quan method [14]. The mean CCI score for BB users was 0.26 with $83.2 \%$ of patients having a CCI score of 0 . The mean CCI score for non-BB users was 0.36 with $77.2 \%$ of patients having a CCI score of 0 . Of the 17 conditions used to derive the CCI, diabetes without chronic complication was the most frequently observed condition in the data with $9.9 \%$ of glaucoma patients having a record related to this diagnosis (see supplementary material for detailed information on comorbidities).

\section{Hospitalizations}

The rate of hospitalization was significantly lower in the patients with a $\mathrm{BB}$ prescription compared to the patients with no BB prescription in the pre-index period $(p=0.004)$. However, the difference between the number of hospitalizations in the $\mathrm{BB}$ user group and the non-BB user group was not significant in the post-index period (Fig. 3).

There was a significant increase in the number of hospitalizations within the BB user group from year prior to year after BB initiation, but not in the non-BB group $(p=0.015)$. There were no statistically significant changes between pre-index and post-index period in

Table 1 Baseline demographics of the CPRD study patients

\begin{tabular}{llll}
\hline Characteristics & Discontinuers & Maintainers & Non-BB users \\
\hline All & 91 & 797 & 7400 \\
$N$ & 57.1 & 49.7 & 52.7 \\
Sex, \% female & $69.4(11.0)$ & $69.4(11.4)$ & $70.7(12.0)$ \\
Age, years [mean (SD)] & & & \\
Age $<65$ years & 34 & 257 & 2091 \\
$N$ & & & 5309 \\
Age $\geq 65$ years & 57 & 540 & \\
$N$ &
\end{tabular}



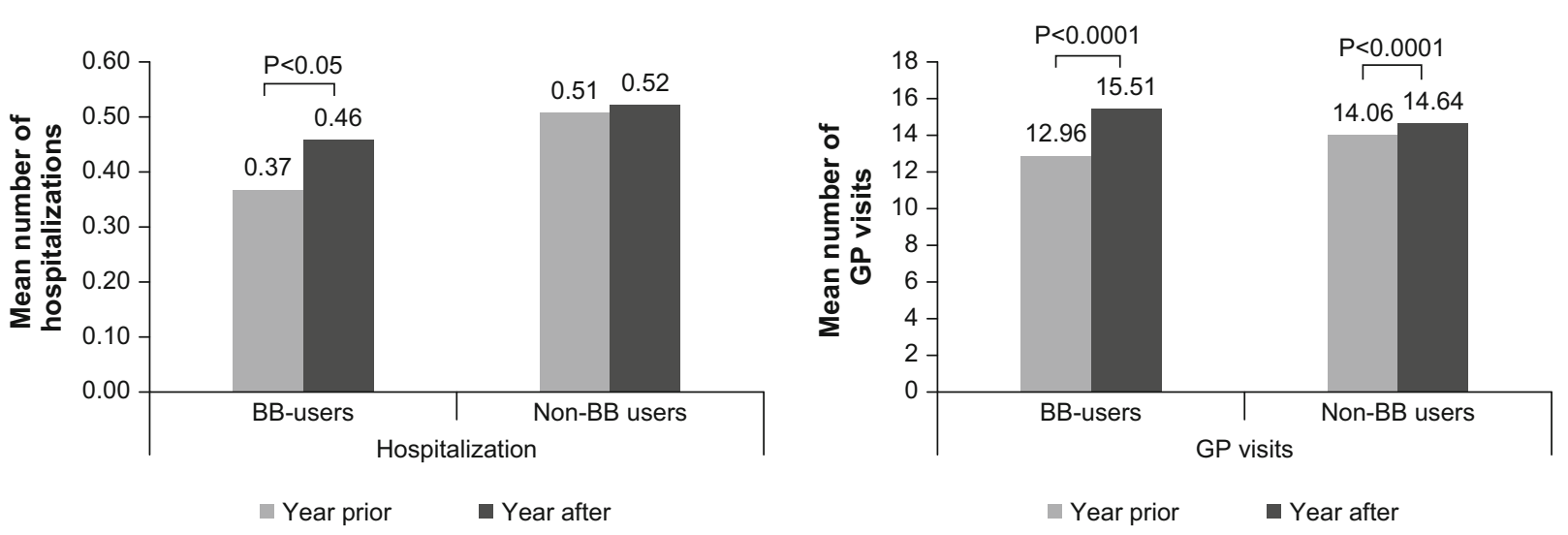

Fig. 3 Mean number of service encounters (hospitalizations and GP visits) per patient in the year prior and the year after the index date for patients prescribed $\mathrm{BBs}$ and patients who were not prescribed BBs

hospitalizations. There was a numerical increase in the average number of hospitalizations in the group of patients that discontinued BB treatment compared to the patients that continued BB treatment. Even within the maintainer group there was a greater increase to the year after BB initiation than in the non-BB user group, although it was not statistically significant (Tables 2, 3).

\section{GP Visits}

There was a very similar pattern in terms of GP visits compared to hospitalizations (Fig. 4). The mean number of GP visits in the 1-year pre-index period was 12.96 for $\mathrm{BB}$ users and 14.06 for non-BB users $(p=0.020)$, and in the 1 -year post-index the mean number of GP visits was 15.51 for $\mathrm{BB}$ users and 14.64 for non-BB users $(p=0.002)$.

\section{Respiratory Events}

In the 1-year pre-index period, $3.9 \%$ of $\mathrm{BB}$ patients and $15.3 \%$ of non-BB patients $(p<0.0001)$ had a diagnosis for respiratory conditions. In the 1-year post-index period, $5.4 \%$ of $\mathrm{BB}$ patients and $16.0 \%$ of non-BB patients $(p<0.0001)$ had a diagnosis for respiratory conditions (Fig. 5). The frequency of

Table 2 Mean number of hospitalizations and GP visits per patient

\begin{tabular}{|c|c|c|c|c|c|c|c|c|c|}
\hline & \multicolumn{3}{|c|}{ Discontinuers } & \multicolumn{3}{|l|}{ Maintainers } & \multicolumn{3}{|c|}{ Non-BB users } \\
\hline & Year prior & Year after & Change & Year prior & Year after & Change & Year prior & Year after & Change \\
\hline \multicolumn{10}{|c|}{ Hospitalization } \\
\hline All & 0.44 & 0.66 & 0.22 & 0.36 & 0.44 & 0.08 & 0.51 & 0.52 & 0.01 \\
\hline$<65$ years & 0.32 & 0.59 & 0.27 & 0.25 & 0.26 & 0.01 & 0.42 & 0.52 & 0.1 \\
\hline$\geq 65$ years & 0.51 & 0.7 & 0.19 & 0.42 & 0.52 & 0.1 & 0.54 & 0.52 & 0.02 \\
\hline \multicolumn{10}{|l|}{ GP visits } \\
\hline All & 15.22 & 19.27 & $4.05^{* *}$ & 12.7 & 15.08 & $2.38^{* * *}$ & 14.06 & 14.64 & $0.58^{* * *}$ \\
\hline$<65$ years & 11.5 & 17.18 & $5.68^{* *}$ & 9.5 & 11.62 & $2.12^{* * *}$ & 10.58 & 10.98 & 0.4 \\
\hline$\geq 65$ years & 17.44 & 20.53 & $3.09^{*}$ & 14.23 & 16.73 & $2.5^{* * *}$ & 15.44 & 16.09 & $0.65^{* * *}$ \\
\hline
\end{tabular}


Table 3 Respiratory and cardiovascular conditions (\%) in cohorts

\begin{tabular}{|c|c|c|c|c|c|c|c|c|c|}
\hline & \multicolumn{3}{|c|}{ Discontinuers } & \multicolumn{3}{|l|}{ Maintainers } & \multicolumn{3}{|c|}{ Non-BB users } \\
\hline & Year prior & Year after & Change & Year prior & Year after & Change & Year prior & Year after & Change \\
\hline \multicolumn{10}{|c|}{ Respiratory disease } \\
\hline All & 6.6 & 14.3 & $7.7^{*}$ & 3.6 & 4.4 & 0.8 & 15.3 & 16 & $0.7^{*}$ \\
\hline$<65$ years & 2.9 & 14.7 & $11.8^{*}$ & 3.5 & 3.5 & 0 & 12.3 & 13.6 & 1.3 \\
\hline$\geq 65$ years & 8.8 & 14 & 5.2 & 3.7 & 4.8 & 1.1 & 16.4 & 16.9 & 0.5 \\
\hline \multicolumn{10}{|c|}{ Cardiovascular disease } \\
\hline All & 26.4 & 31.9 & 5.5 & 22.1 & 24.2 & 2.1 & 24.6 & 23.9 & -0.7 \\
\hline$<65$ years & 5.9 & 8.8 & 2.9 & 14 & 15.6 & 1.6 & 14.6 & 14.5 & 0.1 \\
\hline$\geq 65$ years & 38.6 & 45.6 & 7 & 25.9 & 28.3 & 2.4 & 28.6 & 27.5 & 1.1 \\
\hline
\end{tabular}
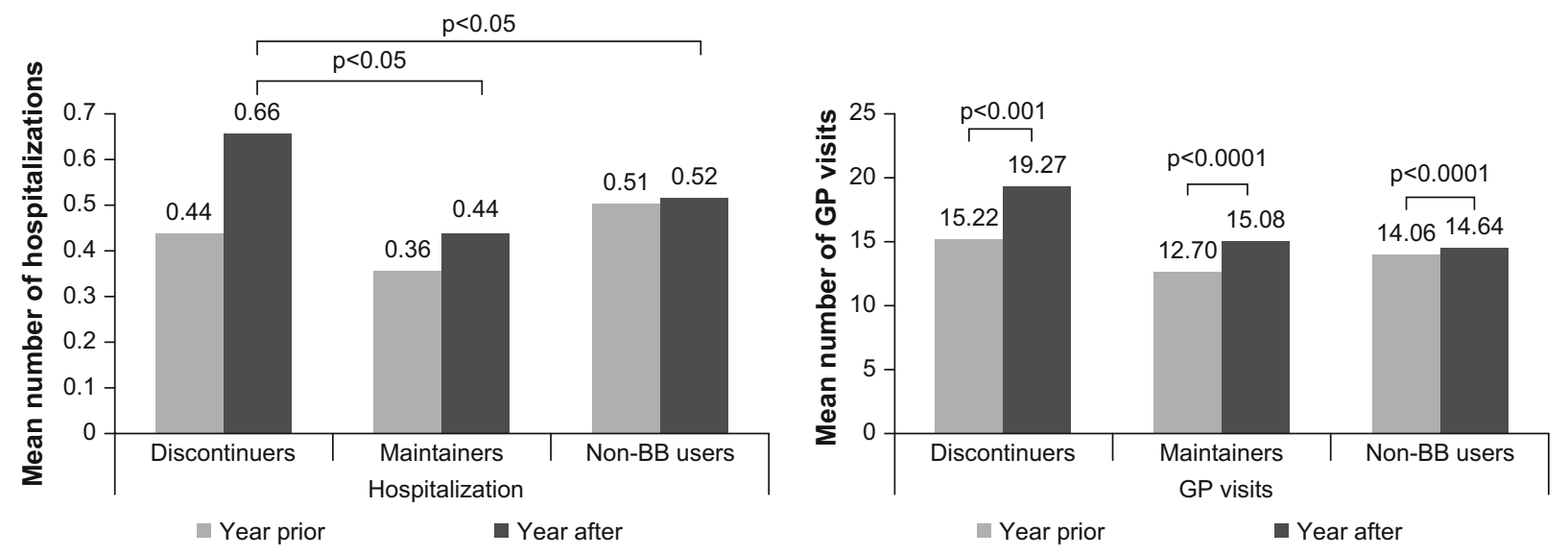

Fig. 4 Mean number of service encounters (hospitalizations and GP visits) per patient in the year prior and the year after the index date

respiratory conditions from the pre- to post-index period increased by $1.5 \%(p=0.037)$ in the BB group, and $0.7 \%(p=0.026)$ in the non-BB group. Both changes were statistically significant, although the increase in the BB user group was greater.

As seen in Fig. 6, patients that discontinued or stayed on $\mathrm{BB}$ had lower proportions of respiratory diagnoses than the non-BB group in the pre-index period. In the post-index period, patients that discontinued $\mathrm{BB}$ use and patients that were non-BB users had a higher proportion of respiratory diagnoses than patients that maintained $\mathrm{BB}$ use. The odds of patients that maintained $\mathrm{BB}$ use having any respiratory condition in the post-index period was significantly lower than patients that discontinued $\mathrm{BB}$ use and patients that were non-BB users (OR 0.249; 95\% CI 0.116, 0.536; $p=0.0004 ;$ and OR $0.203 ;$ 95\% CI 0.132, $0.313 ; p<0.0001)$. There was a statistically significant increase in respiratory event rates post- vs pre-index in patients that discontinued $\mathrm{BB}$ use and patients that were non-BB users but not in patients that maintained $\mathrm{BB}$ use. 

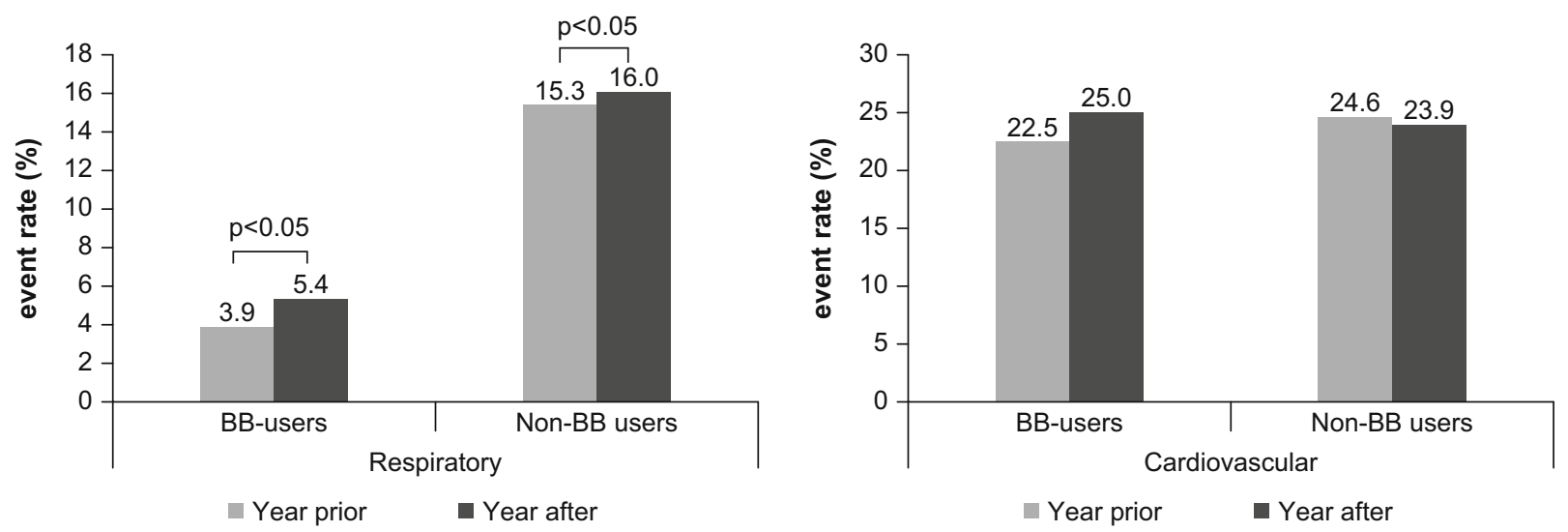

Fig. 5 Respiratory and cardiovascular event rates (\%) in the year prior and the year after the index date for patients prescribed $\mathrm{BBs}$ and patients who were not prescribed $\mathrm{BBs}$
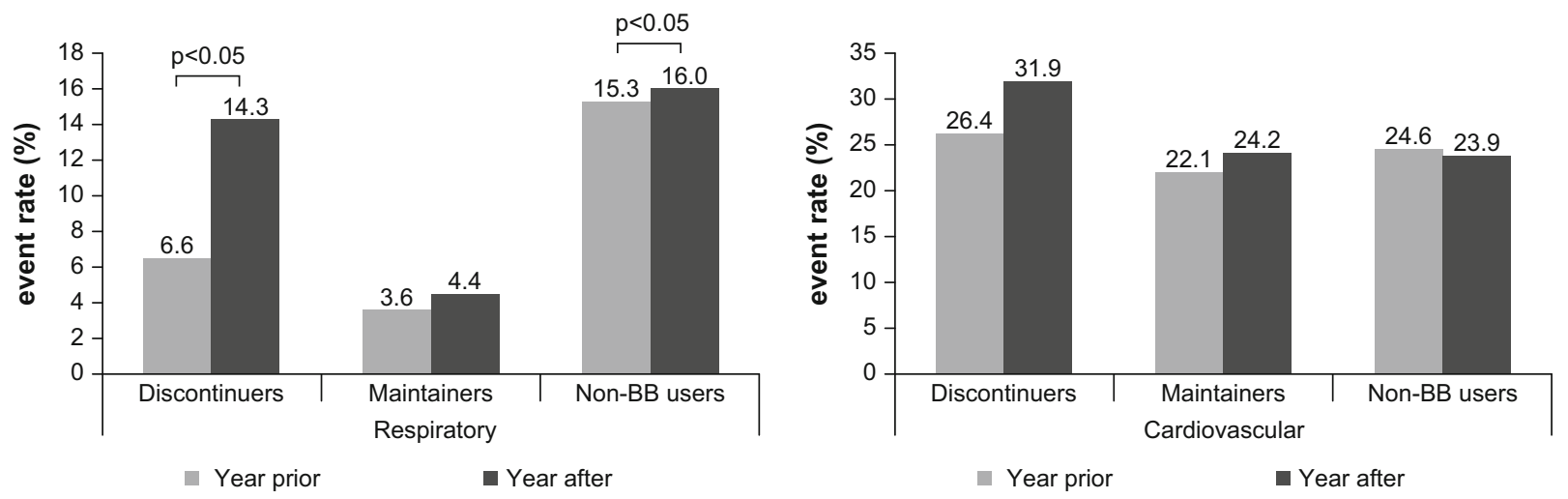

Fig. 6 Respiratory and cardiovascular event rates (\%) in the year before and the year after the index date

\section{Cardiovascular Events}

In the pre-index period, $22.5 \%$ of $\mathrm{BB}$ patients and $24.6 \%$ of non-BB patients had a diagnosis for cardiovascular conditions (Fig. 5). In the post-index period $25.0 \%$ of $\mathrm{BB}$ patients and $23.9 \%$ of non-BB patients had a diagnosis for cardiovascular conditions. Overall there was no statistically significant difference in diagnoses from the pre-index period to the post-index period in either group in terms of any cardiovascular conditions.

There was no significant difference in cardiovascular event rates between groups in the pre-index period. When adjusting for patient characteristics, there was a significant difference in cardiovascular event rates between patients that discontinued $\mathrm{BB}$ use and patients that were non-BB users in the post-index period. In addition, a lower likelihood of having cardiovascular events was found in the post-index period in the patients that maintained BB use vs. patients that discontinued BB use, although the difference is not significant. The odds of patients that discontinued BB use having any cardiovascular conditions in the post-index period was significantly higher than in patients that were non-BB users (OR 1.575; 95\% CI 1.001, 2.479; $p<0.05)$.

\section{Costs}

The mean annual cost of hospitalizations for this glaucoma population was estimated at 
$£ 1876$ for patients that discontinued BB use, $£ 1899$ for patients that maintained BB use, and $£ 1807$ for patients that were non-BB users who had a hospitalization with a valid HRG code in the pre-index period. In the post-index period, the mean annual costs were $£ 2783$ for the patients that discontinued $\mathrm{BB}$ use, $£ 1388$ for patients that maintained $B B$ use, and $£ 2120$ for patients that were non-BB users who had a hospitalization with a valid HRG code in the post-index period.

The mean annual cost of GP visits was estimated to be $£ 694$ for patients that discontinued $\mathrm{BB}$ use, $£ 582$ for patients that maintained BB use, and $£ 651$ for patients that were non-BB users in the pre-index period. In the post-index period mean GP visit cost was $£ 894$ for the patients that discontinued BB use, $£ 690$ for patients that maintained $B B$ use, and $£ 680$ for patients that were non-BB users in the post-index period.

\section{DISCUSSION}

This study shows an increase in the use of medical resources in glaucoma patients in the UK who were initially prescribed beta-blockers but subsequently discontinued their use. Although the results do not evaluate a causal effect, they suggest a potential relationship between use of topical beta-blockers in glaucoma therapy and adverse outcomes.

The rate of hospitalization was significantly lower among patients that used beta-blockers compared to patients that were non-betablocker users in the year prior to beta-blocker initiation, suggesting that healthcare providers are careful about patient selection in respect to when beta-blockers are initiated; those patients who have more hospitalizations are less likely to receive topical beta-blockers in the pre-index period. When beta-blocker users are disaggregated into patients that maintained betablocker use and patients that discontinued beta-blocker use, the significant decrease in hospitalization rate in the year prior is found between the patients that maintained betablocker use and the non-beta-blocker user group, but not between the patients that discontinued beta-blocker use and the nonbeta-blocker user group, perhaps suggesting that there is a subset of patients (discontinuers) for whom beta-blockers were prescribed despite potential contraindications. In the year after being prescribed beta-blockers, the patients that discontinued beta-blockers had significantly higher average number of hospitalizations than patients that maintained beta-blocker use and the non-beta-blocker user group. Similar hospitalization rates were reported by Schmier et al. [12], where discontinuers more than doubled their average rate of all-cause in-patient hospitalizations when years 2 and 3 of the observation period were compared.

The number of GP visits among the patients that discontinued beta-blocker use was not significantly different to the non-beta-blocker user group in the year before being prescribed beta-blockers. In the year after beta-blocker initiation, however, there was a statistically significant difference between all groups, with the greatest number of GP visits being in the patients that discontinued beta-blocker use. The largest increase was observed within the betablocker user group from pre- to post-index and there was a greater increase in the group that maintained beta-blocker use compared to the non-beta-blocker user group as well.

There were lower respiratory event rates in the pre-index period in the beta-blocker user groups (both in patients that maintained beta-blocker use and patients that discontinued beta-blocker use) than in non-beta-blocker users. Patients with pre-index respiratory conditions are less likely to receive beta-blocker therapy than those without pre-index respiratory conditions. The rate of respiratory events in the patients that maintained beta-blocker use did not change much from pre- to post-index period, which may indicate that the respiratory events were mild and the physicians decided to keep the patient on beta-blockers. In the pre-index period, there were fewer respiratory events compared to cardiovascular events. A possible explanation is that, in clinical practice, physicians may be more concerned about respiratory events and, in cases of existing conditions, are less likely to prescribe beta-blockers to those patients. 
Patients that discontinued beta-blocker use and non-beta-blocker users were more likely to have respiratory events post-index versus maintainers. In terms of the post-index events, the most relevant change is the one within the group that discontinued beta-blocker use. There was a statistically significant increase within this group from pre- to post-index, and the post-index event rate approached the one in the non-beta-blocker user group. Although the results indicate that there is a more careful patient selection when prescribing beta-blockers in respiratory (compared to cardiovascular) cases, there still appears to be a certain group of patients for whom beta-blockers are prescribed despite clear contraindication, or in whom significant respiratory adverse events arise despite no clear contraindication.

In the pre-index period, there are larger numbers of cardiovascular events compared to the respiratory events. As discussed, a possible explanation is that there is less concern about cardiovascular contraindications to beta-blockers in prescriptions by physicians (as opposed to concern about respiratory conditions). This potential clinical practice pattern was supported by an additional regression analysis that showed no statistically significant association between beta-blocker prescription and the presence of pre-index cardiovascular conditions. Patients that discontinued beta-blocker use are significantly more likely to have cardiovascular events than non-beta-blocker users in the post-index period, but there was no significant difference in cardiovascular event rates between groups in the pre-index period.

Taken together, the results suggest that cardiovascular conditions may not be the main consideration that physicians have when prescribing beta-blockers. However, there does appear to be a more careful patient selection in terms of pre-existing respiratory conditions. As was the case with Schmier et al. [12], the low percentage of patients that discontinued betablocker treatment may indicate that ophthalmologists may consider co-morbid conditions before prescribing beta-blockers.

As a result of the observational nature of the study, which is based on routinely collected data, there are a number of potential limitations in the analysis and interpretation of these data. The linking of the CPRD database to the HES data for hospitalizations may result in missing data. There may also be some selection bias if factors that affect the prescription of beta-blockers impact the results. For instance, in CPRD, prescription of a drug is used as a proxy for medication use, which may result in overestimations of drug use because it is not possible to determine whether the patient collected their prescription or used their medication. Furthermore, although the GP prescriptions are the data source, the first prescription may have been provided by a hospital not in the CPRD and be missing from this analysis. In this study, there was a relatively low number of patients that discontinued beta-blocker treatment once the exclusion criteria were applied. The number of patients that discontinued beta-blocker use may be underestimated if the patients experienced adverse events soon after taking beta-blockers.

It is important to highlight that the study codes used to identify conditions and comorbidities are determined using subjective medical judgment. In addition, non-beta-blocker users may be overrepresented by patients who are older with a higher number of comorbidities and there may be different comorbidities within specified groups. However, the number of GP visits increased across all patient groups in the post-index period, suggesting that the likelihood of engaging with health care providers was not a significant factor across the groups.

Despite the limitations, this study confirms findings from a previous analysis conducted in the USA, suggesting that careful selection of patients for beta-blocker treatment needs to be performed by the physician given the greater risk of those patients experiencing comorbidities compared to patients not treated with beta-blockers.

\section{CONCLUSION}

The introduction of beta-blockers in a certain group of patients who later discontinue their use is associated with increased use of medical resources (higher number of GP visits and 
hospitalizations) in glaucoma therapy in the UK. The data add to the body of evidence that suggests that the use of topical beta-blockers in glaucoma therapy for patients with respiratory and cardiovascular complications may be associated with adverse outcomes.

\section{ACKNOWLEDGEMENTS}

Alcon Laboratories, Inc., Fort Worth, Texas, USA funded this research, including the journal's article processing charges and Open Access fee. All authors had full access to all of the data in this study and take complete responsibility for the integrity of the data and accuracy of the data analysis. All named authors meet the International Committee of Medical Journal Editors (ICMJE) criteria for authorship for this manuscript, take responsibility for the integrity of the work as a whole, and have given final approval for the version to be published. The authors thank Steven Cartmell (Ph.D.) of Novartis Ireland Limited, Dublin, Ireland for providing medical writing support, which was funded by Novartis Pharma AG, Basel, Switzerland in accordance with Good Publication Practice (GPP3) guidelines (http://www.ismpp. org/gpp3).

Disclosures. Ananth Viswanathan: grants and personal fees from Allergan, personal fees from Alcon, personal fees from Bausch + Lomb, grants from Thea, all outside the submitted work; in addition, Dr. Viswanathan is author of the PROGRESSOR visual field software with royalties paid and is a member and chapter editor of the extant European Glaucoma Society Guidelines Development Task Force. Alberto Ferreira: owns stock and is an employee of Novartis Pharma AG, Basel, Switzerland. Anmol Mullins: owns stock and is an employee of Novartis Pharmaceuticals, USA. Claudio Spera: employee of Novartis Pharma AG, Basel, Switzerland. Paul McDwyer: owns stock and is an employee of Novartis Ireland Ltd, Dublin, Ireland. Eibhlin Hudson: employee of Novartis Ireland Ltd, Dublin, Ireland. David Covert: owns stock and is an employee of Novartis
Pharmaceuticals, USA. Judit Banhazi: employee of Novartis Ireland Ltd, Dublin, Ireland.

Compliance with Ethics Guidelines. This article does not contain any new studies with human or animal subjects performed by any of the authors. The study was approved by the independent scientific advisory committee (ISAC) for CPRD research (Reference No.: 15_093).

Data Availability. Additional data are available upon request to the corresponding author.

Open Access. This article is distributed under the terms of the Creative Commons Attribution-NonCommercial 4.0 International License (http://creativecommons.org/licenses/ by-nc/4.0/), which permits any noncommercial use, distribution, and reproduction in any medium, provided you give appropriate credit to the original author(s) and the source, provide a link to the Creative Commons license, and indicate if changes were made.

\section{REFERENCES}

1. Quigley HA, Broman AT. The number of people with glaucoma worldwide in 2010 and 2020. Br J Ophthalmol. 2006;90:262-7.

2. Bunce C, Xing W, Wormald R. Causes of blind and partial sight certifications in England and Wales: April 2007-March 2008. Eye. 2010;24(11):1692-9.

3. Institute for Quality and Efficiency in Health Care. Factsheet: glaucoma. July 2013. http://www.ncbi. nlm.nih.gov/pubmedhealth/PMH0048141/. Accessed 2 Feb 2015.

4. Bourne RR, Stevens GA, White RA, et al. Causes of vision loss worldwide, 1990-2010: a systematic analysis. Lancet Glob Health. 2013;1:e339-49.

5. Leske MC, Heijl A, Hyman L, Bengtsson B, Dong L, Yang Z. Predictors of long-term progression in the early manifest glaucoma trial. Ophthalmology. 2007; 114:1965-72.

6. Kass MA, Heuer DK, Higginbotham EJ, et al. The Ocular Hypertension Treatment Study: a randomized trial determines that topical ocular 
hypotensive medication delays or prevents the onset of primary open-angle glaucoma. Arch Ophthalmol. 2002;120:701-13.

7. National Institute for Health and Clinical Excellence. CG85: Glaucoma: diagnosis and management of chronic open angle glaucoma and ocular hypertension. April 2009. http://guidance.nice.org. uk/CG85. Accessed 12 Feb 2015.

8. NHS Information Centre for Health and Social Care. Prescription cost analysis 2007. http://www. ic.nhs.uk/statistics-and-data-collections/primary-care/ prescriptions/prescription-cost-analysis-2007. Accessed 10 Feb 2015.

9. Owen CG, Carey IM, De Wilde S, Whincup PH, Wormald R, Cook DG. The epidemiology of medical treatment for glaucoma and ocular hypertension in the United Kingdom: 1994 to 2003. Br J Ophthalmol. 2006;90(7):861-8.

10. Houde M, Castilloux AM, Tingey D, Assalian A, LeLorier J. Prescription of topical antiglaucoma agents for patients with contraindications to beta-blockers. Can J Ophthalmol. 2003;38(6): 469-75.

11. Vinker S, Kaiserman I, Waitman DA, Blackman S, Kitai E. Prescription of ocular beta-blockers in patients with obstructive pulmonary disease: does a central electronic medical record make a difference? Clin Drug Investig. 2006;26(9):495-500.

12. Schmier JK, Covert DW, Lau EC. Medical service encounters and payments associated with topical adjunctive therapy use of timolol for glaucoma. Clin Drug Investig. 2012;32(12):835-42.

13 Tate AR, Beloff N, Al-Radwan B, et al. Exploiting the potential of large databases of electronic health records for research using rapid search algorithms and an intuitive query interface. JAMIA. $2014 ; 21(2): 292-8$.

14 Quan H, Sundararajan V, Halfon P, et al. Coding algorithms for defining co-morbidities in ICD-9-CM and ICD-10 administrative data. Med Care. 2005;43(11):1130-9. 